\title{
Haematological and molecular responses in refractory anaemia with ring sideroblasts and thrombocytosis treated with lenalidomide
}

\section{To the Editor:}

Refractory anaemia with ring sideroblasts and thrombocytosis (RARS-T) is a rare entity and is defined as an overlap syndrome with clinical and morphologic features of both myelodysplastic syndrome (MDS) and BCR-ABL-negative myeloproliferative neoplasm, including marked thrombocytosis associated with abnormal megakaryocytes (1). Mutations in the Janus Kinase 2 gene (JAK2) and/or Splicing Factor $3 B$ subunit 1 (SF3B1) gene have been detected in $50-70 \%$ of patients with RARS-T (2-4). The presence of such a mutation in either $J A K 2$ or $S F 3 B 1$ was associated with a better overall survival in a recent European study (5).

Because of its activity in lower-risk MDS with or without the 5q- cytogenetic abnormality (6) and in myelofibrosis (7), lenalidomide treatment has been given with success in two patients with RARS-T. In this letter, we would like to report a case of RARS-T that was successfully treated by lenalidomide.

An 84-years-old woman was referred for unexplained normocytic anaemia ( $\mathrm{Hb} 7.7 \mathrm{~g} / \mathrm{dL})$ with marked thrombocytosis $\left(1515 \times 10^{9} / \mathrm{L}\right)$. Peripheral blood smear showed a left shift in neutrophils. Bone marrow cytology disclosed hypercellularity (illustrated in Fig. 1A) with $15.6 \%$ erythroid cells, $77.6 \%$ granulopoietic cells, $3.2 \%$ monocytes, $4.0 \%$ lymphocytes, $1 \%$ blasts and numerous megakaryocytes. Ring sideroblasts constituted $90 \%$ of erythroid precursors (Fig. 1B). Erythropoiesis and granulopoiesis were both hyperplasic with evidence of dysplasia. The most prominent feature was the presence of numerous atypical megakaryocytes of different sizes and shapes, many with hypolobated nuclei (Fig. 1C). Metaphase cytogenetics revealed the presence of $5 q-$ in one mitosis, which could not be confirmed by FISH using a specific EGR1(5q31) probe, or Array-CGH analysis (SurePrint G3 Human CGH Microarray). We could not detect any mutation in the $S F 3 B 1$ gene, but the JAK2V617F mutation was retrieved and estimated at $12.5-31 \%$ of JAK2 alleles. Based on these findings, RARS-T with a JAK2-V617F mutation was diagnosed.

Because of her anaemia, cytoreductive treatment was not an attractive option. After transfusion of 4 units of red blood
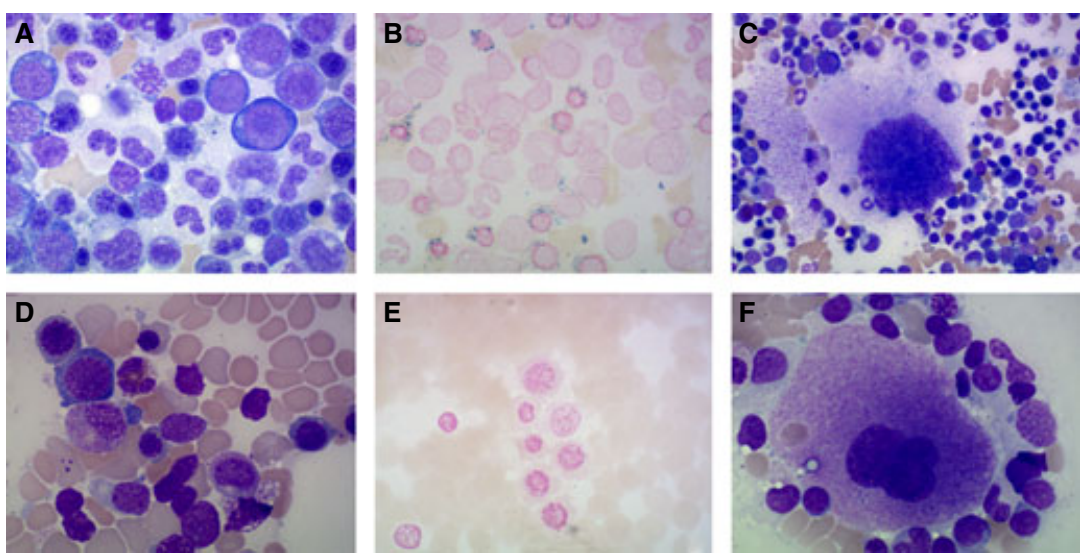

Figure 1 Bone marrow abnormalities before and after treatment with lenalidomide. A-C illustrates the bone marrow abnormalities at diagnosis by applying a May-Grünwald-Giemsa staining on bone marrow smears. (A) Marrow cellularity was increased as a result of erythroid hyperplasia with the evidence of dyserythropoiesis and dysgranulopoiesis. Erythroblasts resembled megaloblasts with nuclear-cytoplasmic maturation with enlarged, lobulated nuclei. Abnormalities of granulocytes included hypogranulation and the acquired pseudo-Pelger-Huët nuclear abnormality. (B) Ring sideroblasts were identified after Prussian blue stain. (C) Some megakaryocytes were dystrophic with a non-lobulated nucleus, while excessive platelet clumps could be identified. D-F illustrates the improvement in myelodysplastic features after lenalidomide treatment. (D) Cellularity in the bone marrow aspirate was normal with normal precursors in granulocytic and erythroid lineages. (E) Prussian blue staining could not retrieve ring sideroblasts. (F) Megakaryocytes recovered their normal morphology with a nucleus containing multiple lobes and a large cytoplasm with numerous purple granules. Pictures were taken using a Zeiss microscope with a Moticam camera (Motic Deutschland, Wetzlar, Germany) (50x and $100 \times$ magnification). 
cells (RBC), the patient was started on lenalidomide $10 \mathrm{mg}$ daily. Her platelet count dropped to $281 \times 109 / \mathrm{L}$, leucocytes normalised, and she became transfusion-independent after 4 months of treatment. After 10 months, the dysplastic hypercellularity (Fig. 1D), atypical megakaryocytes (Fig. 1F) and ring sideroblasts (Fig. 1E) had disappeared from the bone marrow cytology, and the PCR for the JAK2-V617F mutation became negative (detection limit of $2 \%$ mutated $J A K 2$ ). Lenalidomide treatment was continued, but later stopped after 24 months because of asthenia. During the active treatment period, the patient received only one RBC transfusion. The patient is currently followed without active treatment with normal platelet and leucocyte counts and a stable macrocytic anaemia ( $\mathrm{Hb} 9.4 \mathrm{~g} / \mathrm{dL}$ ).

Because of its low incidence, very few clinical studies have been performed in RARS-T. The different treatment options that were reported in a large European series included transfusions and the use of erythropoietin in $50.3 \%$ and $53 \%$ of the patients, the administration of antiplatelet drugs in $51.5 \%$ and cytoreductive therapy in $32.2 \%$ of the cases (8).

Huls et al.(9) have recently published the efficacy of single-agent lenalidomide in two RARS-T patients. Both patients became transfusion-independent after treatment with lenalidomide, one of them had even a molecular response (no detection of JAK2-V617F mutation by quantitative PCR). More recently, lenalidomide was given to a patient with an extensive marrow fibrosis and massive splenomegaly (10). Lenalidomide treatment was able to reduce the splenomegaly, but worsened the various cytopenias. The patient described in this report is a fourth patient, who, besides haematological response, also showed a molecular response.

These case reports indicate that lenalidomide can have beneficial effects for patients with RARS-T. However, retrospective studies on larger cohorts of patients and subgroup analysis in the recent MDS005 trial (comparing lenalidomide with placebo in MDS patients without deletion 5q) focusing on patients with RARS-T are needed to identify the correct place of lenalidomide in patients with RARS-T.

\section{References}

1. Vardiman JW, Bennett JM, Bain BJ, Baumann I, Thiele J, Orazi A. Myelodysplastic/myeloproliferative neoplasms, unclassifiable. In: Swerdlow SH CE, Harris NL, eds. WHO Classification of Tumours of Haematopoietic and Lymphoid Tissues. Lyon: IACR; 2008:85-6.

2. Visconte V, Makishima H, Jankowska A, et al. SF3B1, a splicing factor is frequently mutated in refractory anemia with ring sideroblasts. Leukemia 2012;26:542-5.

3. Malcovati L, Papaemmanuil E, Bowen DT, et al. Clinical significance of SF3B1 mutations in myelodysplastic syndromes and myelodysplastic/myeloproliferative neoplasms. Blood 2011;118:6239-46.

4. Gattermann N, Billiet J, Kronenwett R, Zipperer E, Germing U, Nollet F, Criel A, Selleslag D. High frequency of the JAK2 V617F mutation in patients with thrombocytosis (platelet count $>600 \times 109 / \mathrm{L}$ ) and ringed sideroblasts more than $15 \%$ considered as MDS/MPD, unclassifiable. Blood 2007;109:1334-5.

5. Broseus J, Alpermann T, Wulfert M, et al. Age, JAK2 (V617F) and SF3B1 mutations are the main predicting factors for survival in refractory anaemia with ring sideroblasts and marked thrombocytosis. Leukemia 2013;27:1826-31.

6. List A, Kurtin S, Roe DJ, Buresh A, Mahadevan D, Fuchs D, Rimsza L, Heaton R, Knight R, Zeldis JB. Efficacy of lenalidomide in myelodysplastic syndromes $N$ Engl $\mathrm{J}$ Med 2005;352:549-57.

7. Quintás-Cardama A, Kantarjian HM, Manshouri T, Thomas D, Cortes J, Ravandi F, Garcia-Manero G, Ferrajoli A, BuesoRamos C, Verstovsek S. Lenalidomide plus prednisone results in durable clinical, histopathologic, and molecular responses in patients with myelofibrosis. J Clin Oncol 2009;27:4760-6.

8. Broseus J, Florensa L, Zipperer E, et al. Clinical features and course of refractory anemia with ring sideroblasts associated with marked thrombocytosis. Haematologica 2012;97: 1036-41.

9. Huls G, Mulder AB, Rosati S, van de Loosdrecht AA, Vellenga E, de Wolf JTM. Efficacy of single-agent lenalidomide in patients with JAK2 (V617F) mutated refractory anemia with ring sideroblasts and thrombocytosis. Blood 2010;116:180-2.

10. Taylor G, Culligan D, Vickers MA. Refractory anemia with ring sideroblasts associated with marked thrombocytosis complicated by massive splenomegaly treated with lenalidomide resulting in resolution of splenomegaly but severe and prolonged pancytopenia. Case Rep Hematol 2013;2013:718480.

Jo Caers ${ }^{1}$, Kaoutar Hafraoui ${ }^{1}$, Aurore Keutgens ${ }^{2}$, Jean-Hubert Caberg ${ }^{3}$, Frederic Lambert ${ }^{3}$, Francoise Tassin $^{2}$, Yves Beguin ${ }^{1}$

${ }^{1}$ Department of Hematology, $\mathrm{CHU}$ of Liège, Liège; ${ }^{2}$ Department of Laboratory Hematology, CHU Sart Tilman, Liège; ${ }^{3}$ Department of Genetics, CHU of Liège, Liège, Belgium

Correspondence Dr Jo Caers, MD, PhD, Department of Clinical Hematology, Centre Hospitalier Universitaire de Liège, Domaine Universitaire du Sart Tilman, Bâtiment B 35, B-4000 Liège, Belgium.

Tel: +32 436677 04; Fax: +32 436688 55;

e-mail: jo.caers@chu.ulg.ac.be 